\title{
MENDING TERMINI STATION
}

\section{CERASOLI, Mario}

Universidad Roma Tre

Departamento de Arquitectura

Profesor Asociado

Vía Madonna dei Monti, 40. C.P. 00184 Roma, Italia

Correo electrónico: mario.cerasoli@uniroma3.it

Teléfono: +390657339687

\author{
AMATO, Chiara \\ Sapienza Universidad de Roma \\ Departamento de Planificación, Diseño, Tecnología de la Arquitectura (PDTA) \\ PhD student \\ Via Flaminia 70. Rome, 00196, Italy \\ Correo electrónico: chiara.amato@uniroma1.it \\ Teléfono: +39 3930103943
}

Palabras Clave: remendar; regeneración urbana; movilidad

Key words: mending; urban regeneration; mobility

\section{Resumen}

Mending Termini Station es el título de una experimentación académica que ha sido tratada y profundizada entre 2017 y 2018 en el contexto de un Master en finanzas inmobiliarias (en la LUISS Business School en Roma), un Taller internacional de planificación urbana y arquitectura (con la participación de las universidades de Roma Tre y Sapienza, de Roma, ETSAB/UPC, de Barcelona, UFRGS, de Porto Alegre - Brasil, y UNR, de Rosario - Argentina) y varios cursos de Urbanismo en el Departamento de Arquitectura de la Universidad Roma Tre.

La experimentación se funda sobre la anomalía genética de las ciudades italianas, ligadas al transporte por carretera (auto particulares, buses y camiones), que muestra progresivamente y dramáticamente sus consecuencias, especialmente en la ciudad de Roma - que tiene el territorio municipal más amplio de Europa. El "cuidado del hierro," prometido por el Plan Regulador municipal de 2008, que contemplaba nuevas líneas de metro, tranvías, ferroviarias, corredores preferenciales de transporte público y el cierre del cinturón ferroviario en la parte norte de Roma, lamentablemente sigue apareciendo en las crónicas debido a quiebras y interrupciones.

La congestión del tráfico vehicular, debido a la estructura radial de la red vial romana (que sigue la estructura de las antiguas carreteras consulares), la ineficiencia del transporte público y el flujo monodireccional de viajeros diarios a y del centro de la ciudad, involucra a toda el área metropolitana y paraliza todos los días a los ciudadanos.

En particular, la estructura de los ferrocarriles parece penalizada por el cinturón que todavia nunca se ha completado, por una nueva estación para los trenes de Alta Velocidad, la Estación Tiburtina, subutilizada debido a la presencia dominante de la Estación Termini, la estación principal de Roma, donde llegan la mayoría de los trenes regionales y Alta Velocidad.

Citación: CERASOLI, M. y AMATO, CH. Remendando la estación Termini. En: Libro de proceedings, CTV 2018. XII Congreso Internacional Ciudad y Territorio Virtual. "Ciudades y Territorios Inteligentes". Mendoza, 5-7 septiembre 2018. Barcelona: CPSV, 2018, p. 227-246. 
Por su ubicación, a caballo entre las Murallas Antigua del Emperador Aureliano, que rodean el centro histórico de Roma, la Estación Termini se carateriza como un corte en el medio del corazon de la ciudad, un fragmento urbano que separa los barrios Esquilino, Castro Pretorio, San Lorenzo, y que atrae fenómenos al mismo tiempo de degradación y turistificación masiva.

El proyecto Mending Termini Station está inspirado en una hipótesis del Plan Regulador de 1931 (por el arquitecto Marcello Piacentini). Este Plan, al final de un largo debate que duró más de cincuenta años, propuso la eliminación de la estación de Termini, construida en el cuadrante oriental del centro histórico de Roma, reemplazándola con un ferrocarril subterráneo y una nueva estación ferroviaria, también esta subterránea, y el cierre simultáneo del anillo ferroviario hacia el norte. La zona de Termini, una vez liberada de la infraestructura ferroviaria, estaba destinada a convertirse en un nuevo centro direccional con características monumentales, en línea con lo que se logró en otras capitales europeas.

Mending Termini Station actualiza las ideas de planificación de 1931: la eliminación de la actual estación de Termini, reemplazada por un nuevo trazado ferroviario subterráneo y una nueva estación, también subterranea, únicamente para el Alta Velocidad; la reutilización y valorización de la Estación del 1949 y de su gran área central, liberada de los rieles; el cierre del cinturón ferroviario con la reorganización de la movilidad y del transporte público de toda el área metropolitana de Roma. Todo esto, en total conformidad con las regulaciones europeas sobre la liberalización del mercado ferroviario.

El ensayo ilustrará los estudios preparatorios, los análisis históricos, el modelo actual, las evaluaciones críticas y los métodos de construcción de las propuestas. La metodología de implementación ha previsto un juego de rol, donde estuvieron representados los tres actores principales de la transformación urbana: el Municipio, la empresa que gestiona la red ferroviaria y los edificios ferroviarios, RFI Rete Ferroviaria Italiana (Grupo Ferrovie dello Stato) y finalmente los ciudadanos mismos.

Las soluciones propuestas se caracterizan por su factibilidad urbana, de transporte y movilidad y también socioeconómica. Por lo tanto, constituyen una propuesta sostenible para la ciudad de Roma y su sistema de transporte público.

\section{Abstract}

Mending Termini Station is the title of an academic experimentation that has been treated and deepened between 2017 and 2018 in the context of a Master in Real Estate Finance (at the LUISS Business School in Rome), an international workshop on urban planning and architecture (with the participation of the universities Roma Tre and Sapienza, of Rome, ETSAB / UPC, of Barcelona, UFRGS, of Porto Alegre - Brazil, and UNR, of Rosario - Argentina) and Urban Planning Laboratories at the Department of Architecture of the University "Rome Tre".

The experimentation is based on the genetic anomaly of the Italian cities, linked to road transport (private cars, buses and trucks), that progressively and dramatically shows its consequences, especially in the city of Rome - which has the broadest municipal territory of Europe. The "care of iron", promised by the Municipal Regulatory Plan of 2008, which contemplated new subway lines, trams, railways, preferential corridors of public transport and the closure of the railway belt in the northern part of Rome, unfortunately continues to appear in the chronicles due to bankruptcies and interruptions. 
The congestion of vehicular traffic, due to the radial structure of the Roman road network (which follows the structure of the ancient consular roads), the inefficiency of public transport and the monodirectional flow of commuters to and from the center of the city, involves the entire area metropolitan and paralyzes citizens every day.

In particular, the structure of the railways seems to be penalized by the belt that has not yet been completed, by a new station for high-speed trains, the Tiburtina station, underutilized due to the dominant presence of the Termini station, the main station of Rome, where most of the regional and high-speed trains arrive.

Because of its location, halfway between the Ancient Walls of Emperor Aureliano, which surround the historical centre of Rome, Termini Station is characterized as a cut in the middle of the heart of the city, an urban fragment that separates the Esquilino, Castro Pretorio and San Lorenzo neighborhoods, and that attracts phenomena at the same time of degradation and massive touristification.

The Mending Termini Station project is inspired by a hypothesis of the 1931 Regulatory Plan (by architect Marcello Piacentini). The Plan, at the end of a long debate that lasted more than fifty years, proposed the elimination of the Termini station, built in the eastern quadrant of the historic center of Rome, replacing it with an underground railway and a new railway station, also underground, and the simultaneous closure of the railway ring to the north. The area of Termini, once freed from the railway infrastructure, was destined to become a new directional center with monumental characteristics, in line with what was achieved in other European capitals.

Mending Termini Station updates the planning ideas of 1931: the elimination of the current Termini Station, replaced by a new underground railway line and a new station, also underground, only for High Speed Trains; the reuse and recovery of the 1949 Station and its large central area, freed from the rails; the closure of the railway belt, with the reorganization of mobility and public transport throughout the metropolitan area of Rome. All this, in full compliance with European regulations on the liberalization of the rail market.

This essay will illustrate the preparatory studies, the historical studies, the current model, the critical evaluations and the methods of construction of the proposals. The implementation methodology has planned a role-play, where the three main actors of the urban transformation were represented: The Municipality, the company that manages the railway network and the railway buildings, RFI Rete Ferroviaria Italiana (Gruppo Ferrovie dello Stato) and finally the citizens themselves.

The proposed solutions are characterized by their urban, transport and mobility and also socioeconomic feasibility. Therefore, they constitute a sustainable proposal for the city of Rome and its public transport system.

\section{El Workshop}

Mending Termini Station es el título de una experimentación académica que nació casi como un juego y luego se convirtió en un tema de investigación proyectual que se basa en la anomalía genética de las ciudades italianas relacionada con la movilidad por carretera, que está mostrando, progresivamente y dramáticamente, sus consecuencias, especialmente en Roma.

Citación: CERASOLI, M. y AMATO, CH. Remendando la estación Termini. En: Libro de proceedings, CTV 2018. XII Congreso Internacional Ciudad y Territorio Virtual. "Ciudades y Territorios Inteligentes". Mendoza, 5-7 septiembre 2018. Barcelona: CPSV, 2018, p. 227-246. 
Al principio, el tema fue tratado y profundizado durante el curso de Urbanismo y Planificación Territorial del MAREF, Master en Finanzas Inmobiliarias (en la Escuela de Negocios LUISS de Roma) en marzo de 2017; luego en el Workshop Roma 2017 "Mending Termini Station", taller internacional de urbanismo y arquitectura", en septiembre de 2017; en el Laboratorio de Planificación Urbana, en el tercer año de la licenciatura en Ciencias de la Arquitectura, entre octubre de 2017 y febrero de 2018, y en el Curso de Proyecto de Recuperación Urbanistica, abierto a estudiantes de las tres maestrías en Arquitectura, del Departamento de Arquitectura de la Universidad Roma Tre, entre marzo y julio de 2018. Finalmente, fue objeto de una tesis de grado, discutido en julio de 2018 (por el Dr. Arturo Becchetti y el Dr. Fabricio Felici).

Durante el Workshop, se implementó una metodología de juego de roles, representando a los tres actores principales de la transformación urbana:

- el Municipio de Roma;

- la empresa que gestiona la red ferroviaria y los inmuebles de los ferrocarriles (RFI Rete Ferroviaria Italiana, perteneciente al Holding Ferrovie dello Stato);

- los ciudadanos involucrados en la transformación.

The various solutions proposed are characterized by urban, transport and socio-economic feasibility. Therefore, they constitute a feasible and sustainable integrated proposal for the city of Rome and its public transport system.

\subsection{La movilidad romana}

La movilidad romana sufre de males antiguos. El tráfico de automóviles está constantemente congestionado en el área metropolitana y paraliza a cientos de miles de personas cada día ${ }^{2}$. Esto se debe tanto a la estructura radial característica de la red de carreteras romanas (derivada de las antiguas carreteras consulares de la antigua Roma), como a la falta de oferta y la ineficiencia del transporte público ${ }^{3}$ y a la concentración de funciones en las áreas centrales y en particular en el centro de la ciudad, que condiciona los flujos de cercanías provenientes de toda la Ciudad Metropolitana y más allá.

De hecho, los trenes de alta velocidad y, sobre todo, la mayoría de los trenes regionales llegan a la estación de Termini, donde se atienden a 500,000 pasajeros al día, la mayoría de los cuales viajan diariamente (por trabajo y estudio) pero también turistas. Debido a su posición, en el centro histórico de Roma, la estación Termini representa hoy un "cortocircuito" dentro de la ciudad, un corte que realmente separa los barrios Esquilino, Castro Pretorio y San Lorenzo, donde degradación y, al mismo tiempo, fenómenos de turistificación salvaje son concentrados.

La "cura del hierro" prometida por el Plan Municipal de 2008, que contemplaba nuevas líneas de metro, tranvía, ferrocarril, nuevos corredores de transporte público y el cierre del cinturón ferroviario en el norte de Roma continúa apareciendo en las noticias solo por accidentes, interrupciones en el servicio, quiebras, consultas judiciales.

\footnotetext{
${ }^{1}$ En el workshop han participado estudiantes y profesores de las escuelas de arquitectura de las universidades Roma Tre y Sapienza, de Roma, de la Universidad Politécnica de Cataluña, de Barcelona, de la Universidad Nacional de Rosario, Argentina, y de la Universidad Federal de Rio Grande del Sur, de Porto Alegre, Brasil.

2 Según los datos Aci, un romano pasa tres días al año en tráfico.

${ }^{3}$ El transporte público en Roma (2.873.494 habitantes en 2016) se lleva a cabo por 2052 autobuses, 30 trolebuses y 164 tranvías (1.279 habitantes / automóvil) más 105 trenes de las tres líneas de metro y 68 trenes ferroviarios regionales otorgados en un Red que en total alcanza los $105 \mathrm{~km}$ de extensión. Para comparar, a Milán viajan 1334 autobuses, 137 trolebuses y 493 tranvías (688 habitantes / automóvil), más 170 trenes de solo 4 líneas de metro en una red de $97 \mathrm{~km}$ (a esto se debe agregar la red ferroviaria RFI y Trenord). 
Figura 1. Desplazamientos diarios en el Municipio de Roma

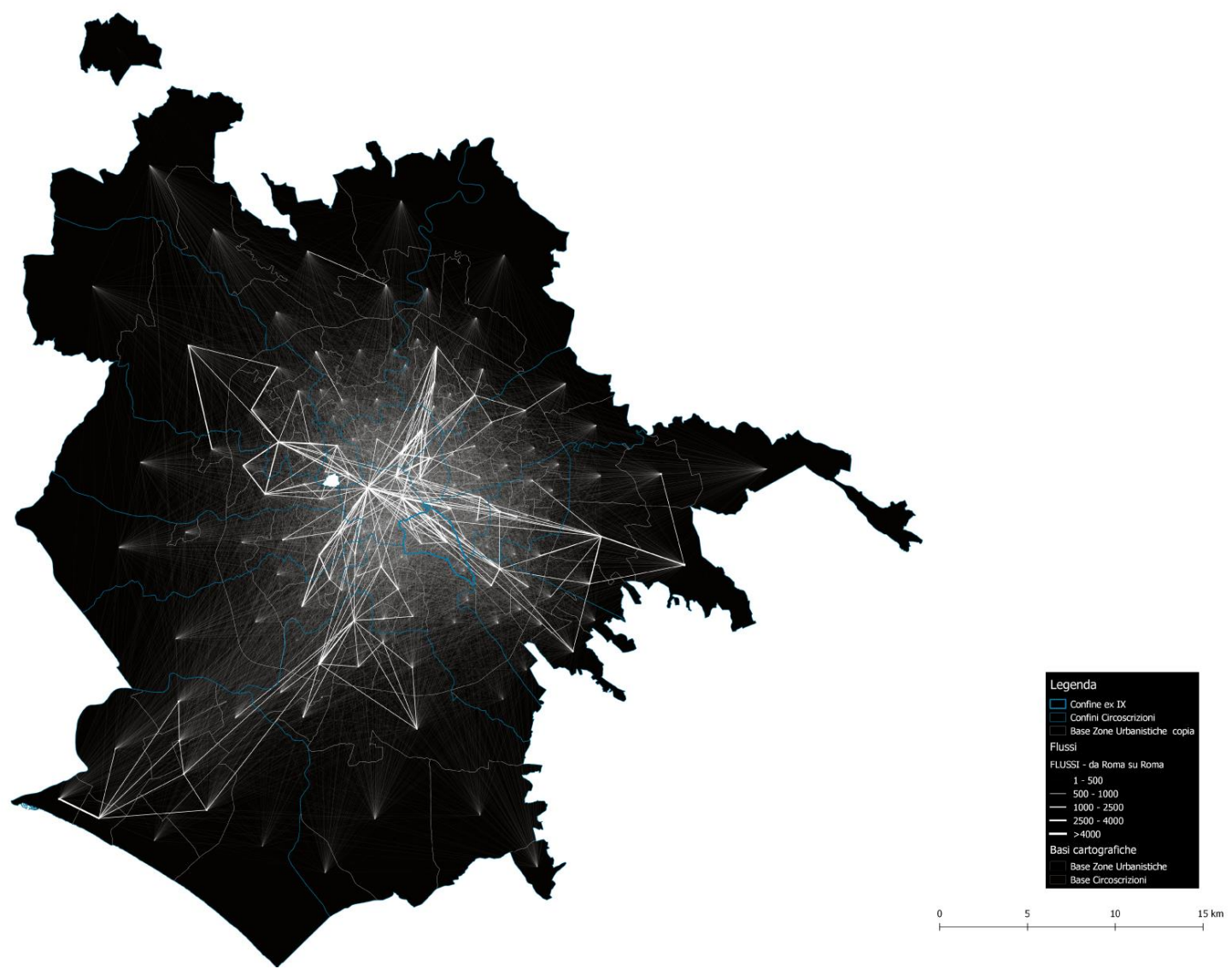

Fuente: Estudio desarrollado por la Dra. Silvia Giachetti. [slv.giachetti@gmail.com], 2018. Universidad Roma Tre.

El escenario Mending Termini Station se inspira en una hipótesis del Plan municipal de 1931 (M. Piacentini). Al final de un largo debate que duró más de cincuenta años, el Plan preveía la eliminación de la Estación Termini, ubicada en el cuadrante este del Centro Histórico de Roma, reemplazándola con un enlace ferroviario subterráneo y una nueva estación central, y con el cierre simultáneo del Anillo Ferroviario al norte. El área de Termini, una vez liberada de la infraestructura ferroviaria, se destinaba a convertirse en un nuevo centro direccional con características monumentales, en línea con lo que se logró hacer en otras capitales europeas.

Mending Termini Station actualiza las soluciones del Plan 1931 y prevé:

- el cierre del Anillo Ferroviario y la finalización de la red de metropolitanas (ya programada);

- la construcción de un túnel ferroviario subterráneo y una nueva estación Termini subterránea dedicada únicamente a los trenes de Alta Velocidad (reentra la actual estación histórica se conserva en la superficie);

- una valoración inmobiliaria de parte del área ferroviaria anterior, para compensar los costos de la operación general;

- la producción de "ciudad pública" (áreas peatonales, áreas verdes públicas, servicios, instalaciones y equipamientos públicos, etc.);

- la mejora de la movilidad urbana (permitida por la eliminación de la actual "barrera" ferroviaria) y la regeneración urbana del área alrededor de la estación.

Citación: CERASOLI, M. y AMATO, CH. Remendando la estación Termini. En: Libro de proceedings, CTV 2018. XII Congreso Internacional Ciudad y Territorio Virtual. "Ciudades y Territorios Inteligentes". Mendoza, 5-7 septiembre 2018. Barcelona: CPSV, 2018, p. 227-246. 
Este artículo ilustra los estudios preparatorios, el análisis histórico, el modelo urbano actual, las evaluaciones de la viabilidad socioeconómica y urbanística del escenario Mending Termini Station.

\section{Estaciones y zonas ferroviarias, patrones de movilidad, liberalización del mercado ferroviario y regeneración urbana: una reseña de experiencias.}

La relación entre ferrocarriles y liberalización del mercado ferroviario, movilidad y regeneración urbana es compleja, pero al mismo tiempo muy estrecha y tiene sus orígenes en los últimos 60 años.

La ciudad contemporánea hoy aparece caracterizada por fenómenos que se repiten en todas las latitudes, como la expansión sin límites de la ciudad y la dispersión de los asentamientos, la desaparición de la separación entre ciudad y campo, resultado de procesos espontáneos y el cambio de algunos patrones del habitar que han marcado la reciente fase de crecimiento de la ciudad.

En las últimas décadas, la polarización en las grandes ciudades, donde se han concentrado las principales ofertas de empleo y servicios públicos, junto con políticas que han penalizado efectivamente las actividades agrícolas, ha fomentado el abandono de los centros menores y territorios internos a favor de las áreas urbanas, grandes y menos grandes, donde, con la urbanización de las áreas periféricas, se produjo el fenómeno de la suburbanización (que Giancarlo De Carlo llamó "ciudad-región" en la década de 1960), ese territorio metropolitano, fragmentado y generalizado, dependiente por dinámicas económicas y de movilidad diaria desde el gran núcleo urbano principal, y que existe como "ciudad del automóvil" (Indovina, 2009).

El fenómeno de la suburbanización estuvo acompañado, en una relación de causa y efecto, por un empeoramiento progresivo de la capacidad y la eficiencia del transporte público, subordinado jerárquicamente al transporte privado por carretera, que en cambio se vinculó estrechamente con este fenómeno. Las políticas territoriales y de movilidad y transporte han comenzado a separarse entonces en muchas ciudades italianas, incluida Roma.

Esto también porque, en comparación con otras ciudades europeas, las italianas se desarrollaron tarde, con la gran expansión urbana ocurrida después de la Segunda Guerra Mundial, cuando la motorización masiva ya estaba explotando (Campus Venuti, 2001).

La política italiana, siguiendo modalidades similares en muchos otros países occidentales y especialmente latinos, pero no solo, ha elegido el transporte privado por carretera como solución moderna para la movilidad, sin evaluar las consecuencias que esto habría tenido sobre el territorio y las ciudades, especialmente las ciudades históricas, incompatibles con el automóvil.

El vaciamiento de territorios enteros, el abandono de los centros históricos menores en favor de la ciudad difusa, fácilmente accesible a través de las nuevas infraestructuras viales (autopistas), son al mismo tiempo efectos y causas de un enorme cambio socioeconómico y ambiental que ahora ha mostrado su insostenibilidad bajo muchos puntos de vista. El consumo

Citación: CERASOLI, M. y AMATO, CH. Remendando la estación Termini. En: Libro de proceedings, CTV 2018. XII Congreso Internacional Ciudad y Territorio Virtual. "Ciudades y Territorios Inteligentes". Mendoza, 5-7 septiembre 2018. Barcelona: CPSV, 2018, p. 227-246. 
de suelo, típico del sprawl, además de erosionar las tierras cultivables, a menudo de calidad (como ocurre, por ejemplo, en la ciudad de Mendoza en Argentina, en detrimento de las culturas vitivinícolas de prestigio), se basa exclusivamente en la movilidad privada individual. Esto se traduce en mayores emisiones de $\mathrm{CO} 2$, con enormes costos para los ciudadanos y sobre todo para la comunidad y para la reducción de la calidad de vida. El $80 \%$ de las actividades diarias de la ciudad implican hoy la necesidad de moverse, en gran parte una "movilidad inútil y obligatoria" (Cerasoli, 2015) que influye en la libertad urbana y la calidad de vida (varios estudios recientes muestran la relación social y económica insostenible entre los costos de salud pública y el uso del transporte privado).

El de la movilidad es evidentemente un tema que afecta transversalmente a todos los territorios que pueden definirse como "urbanizados", desde las áreas internas hasta las grandes ciudades y sus cinturones periféricos.

En el territorio italiano no hay duda de que la red ferroviaria se ha visto afectada por estas dinámicas y ha sufrido elecciones políticas y económicas y procesos sociales que han llevado a los pasajeros a otros modos de movilidad, lo que ha provocado la eliminación progresiva de muchas líneas. Una dinámica que se ha puesto en marcha desde principios de los Cincuenta, cuando, con la construcción de la Autopista del Sol (Autostrada del Sole, 1956-1964), se decidió favorecer la movilidad motorizada en lugar del transporte público por ferrocarril.

La política de liberalización del transporte ferroviario europeo, que comenzó en la década de 1990 con el objetivo de relanzar el fragmentado mercado ferroviario europeo, ha modificado aún más las cuencas de movilidad e influido en la calidad de los servicios y el desarrollo de redes. Mientras que dentro de las ciudades ha tenido fuertes consecuencias en las políticas de transformación de áreas y edificios ferroviarios (Pucci, 2012).

Es la movilidad, en particular la movilidad pública, el sistema que impulsa la ciudad. Las áreas más fragmentadas de la ciudad contemporánea a menudo deben su condición a una movilidad ineficaz $y$, a veces, contraproducente.

Si hablamos de infraestructuras y fragmentación, hablamos de la estructura de la movilidad, de los nodos de intercambio, de las relaciones con las centralidades, los espacios públicos y los lugares centrales conectados a las estaciones, de conexión en el sentido de llevar de un punto a otro y conexión en el sentido de mantener juntas las partes de la ciudad, funcionando y cohesionadas.

Un sistema de movilidad pública caracterizado por infraestructuras escasas o incluso incompletas, por unos pocos nodos de interconexión intermodales, congestionados y mal posicionados, líneas de ferrocarril en desuso y falta de coordinación entre los diferentes sistemas de movilidad tiene repercusiones negativas en todo el territorio.

Y Roma es uno de los casos de este mal funcionamiento.

La planificación urbana de los tres primeros cuartos del siglo pasado tuvo la tarea de construir la ciudad, de expandirla y de planificar equipos e infraestructuras para los nuevos habitantes. Durante más de un cuarto de siglo, la planificación urbana se ha enfrentado a un cambio en las formas de los modos de crecimiento de las ciudades y, durante casi una década, a una crisis inmobiliaria que a menudo produce abandono y desuso.

Citación: CERASOLI, M. y AMATO, CH. Remendando la estación Termini. En: Libro de proceedings, CTV 2018. XII Congreso Internacional Ciudad y Territorio Virtual. "Ciudades y Territorios Inteligentes". Mendoza, 5-7 septiembre 2018. Barcelona: CPSV, 2018, p. 227-246. 
La desindustrialización y los cambios en los modelos económicos han llevado a la producción de áreas en desuso, fragmentos urbanos dentro de la ciudad, antiguamente áreas de producción de todo tipo, hoy elementos de degradación que atraen a otros elementos de degradación, llevando las características de la periferia degradada al centro de la ciudad, con problemas físicos (de permeabilidad urbana) y problemas sociales (de desintegración del tejido social de barrio).

El remiendo de estos fragmentos recuerda la necesidad de regeneración urbana, ese complejo de acciones destinadas a mejorar aquellas partes de las ciudades existentes que han sido degradadas o cuyas funciones anteriores han cesado y son críticas.

En tiempos de crisis de la producción de las construcciones, la regeneración se está convirtiendo cada vez más en un escenario privilegiado de intervención en la ciudad contemporánea. Por lo tanto, las intervenciones de regeneración urbana deben ir inevitablemente más allá de las tendencias del mercado inmobiliario, aún demasiado ligadas a los modelos de asentamiento "dispersos", anticipando las opciones de sostenibilidad que ya se requieren hoy: la reducción del consumo de suelo y la recuperación de la "calidad urbana" (Cerasoli, 2018).

Actualmente, en algunas ciudades italianas, como Milán, Turín, Génova, Bolzano, se ha puesto en marcha la reutilización de las zonas ferroviarias en desuso - fragmentos urbanos en las zonas centrales de la ciudad - a la luz de las experiencias adquiridas en Europa entre los años Noventa y Dos Mil.

Hay varios ejemplos que pueden citarse: desde las intervenciones para reparar ciudades en los lugares donde se encuentran sedimentos ferroviarios abandonados (ZAC Rive Gauche en París en los años 90, Parque Görlitzer en Berlín en los años 70) o para la reorganización de las estaciones (Gare Montparnasse en París en los años 60, King's Cross en Londres en el '96) hasta las reutilizaciones de las estaciones (Museè d'Orsay, París, Hamburger Bahnhof en Berlín en el '96), o las líneas en desuso (Promenade Platée en París '94).

Incluso en Italia, entre los años Ochenta y Noventa, las principales ciudades han visto intervenciones o propuestas, en algunos casos especulativas, sobre áreas en desuso (industriales o ferroviarias): en Milán, las antiguas áreas industriales de Alfa Romeo, Pirelli , Montedison, las propiedades municipales frente a la estación Garibaldi y el área de la estación Milan Porta Nuova; en Turín, las zonas Fiat de Lingotto y Mirafiori y las del Passante Ferroviario ("Espina de Turín"); en Nápoles, las áreas públicas y privadas de Poggioreale, Ilva di Bagnoli, Ponticelli; en Génova, las áreas de S. Benigno, Corte Lambruschini y del puerto; en Florencia, la antigua zona de Fiat en el barrio de Novoli y en Ravenna, las zonas de la antigua "Darsena Petroli".

La oportunidad que hoy se deriva de estas áreas en proceso de regeneración está representada por múltiples elementos que contribuyen a que sean la ocasión para nuevos debates disciplinarios, políticos y procedimentales. En primer lugar, sus posición central dentro de la ciudad consolidada - a menudo adyacentes o, como en el caso del área de Termini en Roma que veremos más adelante, en el interior de la ciudad histórica - resultado del crecimiento urbano que ha incorporado estas zonas, una vez periféricas; el alto grado de conectividad de estas en el marco de las redes de movilidad, ya que formaban parte de ellas antes del abandono; y, finalmente, la complejidad del estatus legal de las compañías ferroviarias. Todos son elementos que hacen de las áreas ferroviarias en desuso una categoría

Citación: CERASOLI, M. y AMATO, CH. Remendando la estación Termini. En: Libro de proceedings, CTV 2018. XII Congreso Internacional Ciudad y Territorio Virtual. "Ciudades y Territorios Inteligentes". Mendoza, 5-7 septiembre 2018. Barcelona: CPSV, 2018, p. 227-246. 
atractiva que necesita acuerdos que involucran una variedad de actores públicos y privados, pero que, si no se gestionan correctamente, también representan un gran riesgo para la especulación inmobiliaria que pueda surgir y para los procesos de gentrificación o formación de guetos que se pueden poner en marcha.

\section{Antecedentes: el Plan de 1931 y la actualidad de su hipótesis}

Desde su construcción en 1874, los efectos de la Stazione Termini sobre el contexto urbano circundante, dentro de las antiguas murallas de la Roma imperial, originan un largo debate sobre su insuficiencia como estación central y la necesidad de su deslocalización. A este debate sigue una serie de propuestas que luego desembocan en el Plan de 1931 elaborado por Marcello Piacentini con Gustavo Giovannoni y Antonio Muñoz.

"Una intervención muy importante en la estrategia general del Plan fue la nueva disposición de la red ferroviaria. Desarrollando una idea ya expuesta por Piacentini en 1926 y retomada en el Piano del GUR ${ }^{4}$, se previó la eliminación de la estación de Termini y su reemplazo por dos nuevas estaciones: la Flaminia al norte, cerca del campo Parioli y la Casilina al sur, a la altura del Mandrione. En la primera llegaban las líneas del sur (Sulmona y las dos para Nápoles), en la segunda las del norte (Florencia, Ancona, Pisa). El tramo urbano de conexión entre estas dos estaciones era subterráneo y tenía su salida principal en Termini, que por lo tanto mantuvo su papel como importante nodo de distribución, pero ya no en la superficie. De esta manera, asumiendo siempre el esquema propuesto por el GUR, era posible, en el área anteriormente ocupada por las vías del tren, construir un nuevo centro urbano, ubicado en una gran avenida de 100 metros de ancho y dividida en tres cuadrados, que conectaban Plaza Esedra con Porta Maggiore. Esta intervención habría permitido un cambio efectivo del centro de gravedad de la ciudad hacia el este, un cuadrante en el que el Plan preveía concentrar la mayor expansión"5.

La hipótesis del Plan de 1931 estaba en línea con lo que ya estaba sucediendo en otras grandes ciudades europeas - la adaptación de la red ferroviaria a las necesidades cambiantes del crecimiento urbano - y se basaba en dos intervenciones estructurales: el cierre del cinturón ferroviario, que desde entonces habría tomado el nombre de "Anillo Ferroviario"; y la eliminación de la Estación Termini, reemplazada por una estación subterránea a lo largo de una nueva vía de tren, también subterránea, que habría cruzado la ciudad de norte a sur.

La nueva estructura ferroviaria conducía a un escenario más funcional y adecuado para la ciudad de Roma, para el cual el Plan había previsto de manera realista la duplicación de la población en un período de 25 años (la duración legal del instrumento de planificación urbana), pasando de poco menos de un millón de habitantes (930.723 al Censo de 1931) a dos millones (y al Censo de 1961, los habitantes eran 2.187.682).

Se le encomendó al "Anillo del ferrocarril" la tarea de conectar las estaciones entonces existentes desde la estación de carga de Nuovo Salario hasta la estación San Pietro, a lo largo de las líneas Roma-Florencia, Roma-Civitavecchia y Roma-Viterbo, cerrando el tramo norte desde San Pietro hasta Nuovo Salario y con la construcción de una nueva estación Flaminia al

\footnotetext{
${ }^{4}$ El GUR Gruppo Urbanisti Romani fue un grupo de arquitectos romanos activos entre los años veinte y treinta. Incluía a Luigi Piccinato, Gino Cancellotti, Giuseppe Nicolosi, cesare Valle y Marcello Piacentini

${ }^{5}$ Fuente: ROSSI, P.O. (1991); Roma. Guida all'architettura moderna 1909-1991. Laterza, Bari. Pagg. 65-68.
}

Citación: CERASOLI, M. y AMATO, CH. Remendando la estación Termini. En: Libro de proceedings, CTV 2018. XII Congreso Internacional Ciudad y Territorio Virtual. "Ciudades y Territorios Inteligentes". Mendoza, 5-7 septiembre 2018. Barcelona: CPSV, 2018, p. 227-246. 
norte, en la que habría atestiguado el enlace ferroviario que la conectaba al sur con la nueva estación Casilina, en el distrito del Mandrione, a lo largo de la línea Roma-Nápoles.

De hecho, ya se pensaba utilizar la red ferroviaria como red metropolitana, para reducir la brecha que separaba Roma desde otras grandes ciudades europeas como París, Londres, Madrid, Berlín, Barcelona.

A la red ferroviaria habría sido agregada luego la vasta red de tranvías de Roma, que, incluso después de la Reforma de 1930, contaba 53 líneas para un total de $350 \mathrm{~km}$ de operativo y más de 100 de red, dando lugar así a un sistema de transporte público eficaz para esos tiempos.

Al mismo tiempo, la intervención urbana en el área ferroviaria de Termini tendría que dar lugar a una intervención de remiendo urbano radical, con la creación de un "sistema direccional central", estructurado en una gran avenida que habría conectado la Piazza di Porta Maggiore con Piazza Esedra y permitiendo la conexión entre los barrios Esquilino, San Lorenzo y Castro Pretorio.

Desafortunadamente, la propuesta del Plan Piacentini no recibió los favores del entonces liderazgo de Ferrovie dello Stato, que tenía muchas dudas sobre la construcción de la línea subterránea y, sobre todo, la nueva estación subterránea. Y lograron convencer a Mussolini, quien, aprobando el Plan municipal, canceló solo el proyecto para la Estación Termini. Perdiendo así una oportunidad preciosa para alinear Roma con las otras ciudades europeas importantes.

\section{Figura 2. Plan de Roma 1931 (M. Piacentini)}

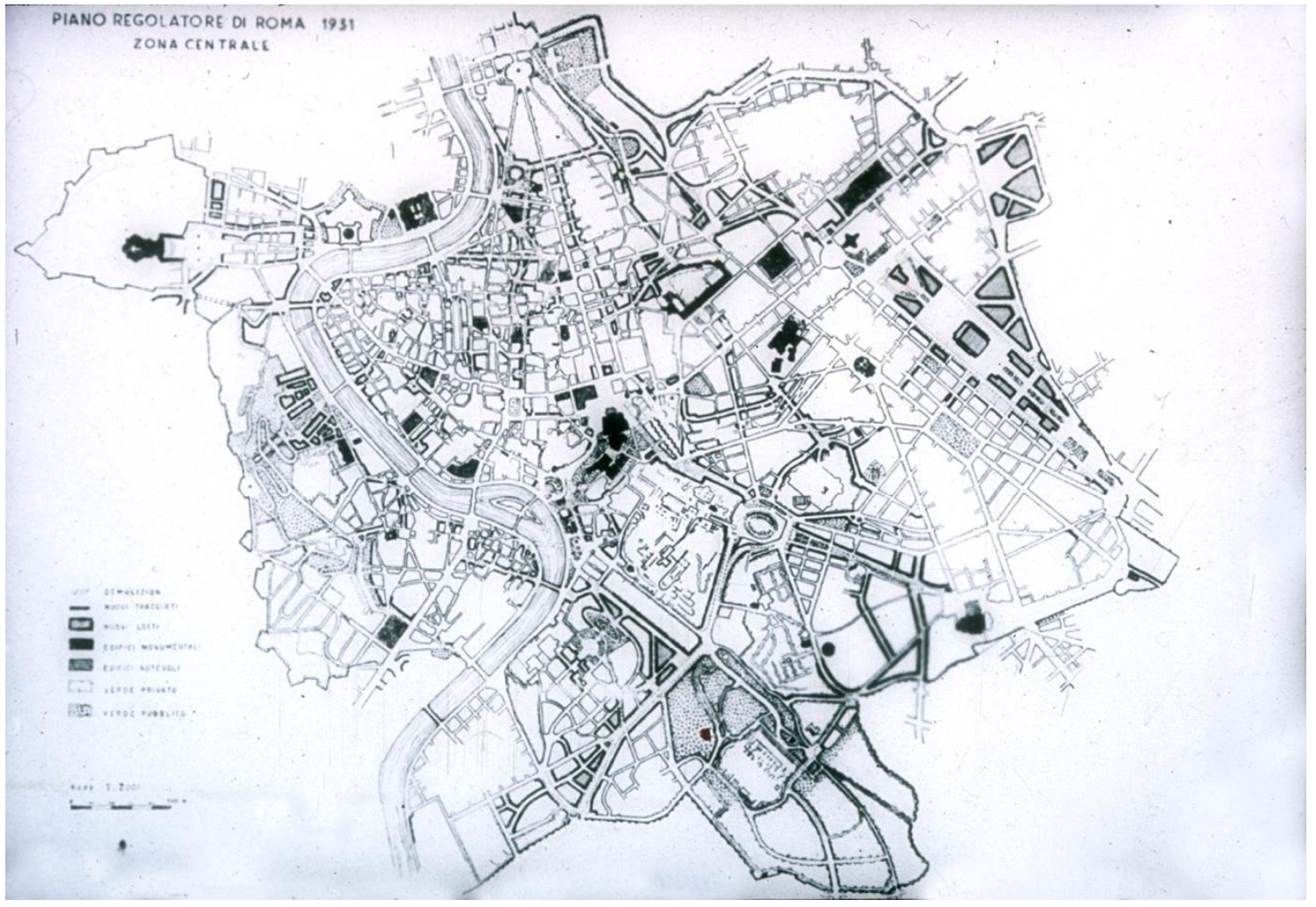

Fuente: Comune di Roma

Citación: CERASOLI, M. y AMATO, CH. Remendando la estación Termini. En: Libro de proceedings, CTV 2018. XII Congreso Internacional Ciudad y Territorio Virtual. "Ciudades y Territorios Inteligentes". Mendoza, 5-7 septiembre 2018. Barcelona: CPSV, 2018, p. 227-246. 


\section{Infraestructuras, movilidad y planificación en Roma: ¿una relación conflictiva?}

Antes del Plan de 2008, los únicos planes urbanísticos de Roma que han tratado el tema de la movilidad y el sistema de infraestructura han sido el de 1909, que trazó la red del tranvía, y el de 1931, con la hipótesis del "Anillo del ferrocarril" y el proyecto para Termini. Sin embargo, incluso entonces la relación entre planificación y movilidad mostró importantes grietas, tanto que el ambicioso proyecto para Termini se eliminó del Plan 6 .

En los últimos años del fascismo, primero la guerra en Etiopía de 1935-36 y luego la entrada de Italia en la Segunda Guerra Mundial retrasó la implementación del Plan de 1931, así como de todas las demás obras "fuera de plan" planificadas a lo largo de los años. Como el nuevo distrito para el E42, la Exposición Universal de Roma en 1942, que nunca se celebró, pero que dejó un barrio fantasma y, casi completa, la primera línea del metro de Roma, que debía conectarse a la estación Termini.

Para la Estación Termini, se inició el programa para reemplazar el edificio previo diseñado por Salvatore Bianchi, en 1874, con la asignación del proyecto para una estación más grande al Ing. Angiolo Mazzoni. Sin embargo, las obras, ya iniciadas, se bloquean en 1943, dejando el edificio sin la fachada principal ${ }^{7}$.

En la inmediata Posguerra, cuando fue necesario emplear a un gran número de personas en actividades laborales para reiniciar la economía del país, se reanudaron también los trabajos para completar la construcción del "Anillo Ferroviario", según el Plan de 1931. Sin embargo, hacia mediados de los años Cincuenta, debido a los cambios en las condiciones económicas de la ciudad de Roma y de Ferrovie dello Stato, se suspendieron nuevamente, esta vez definitivamente, y el trazado, a lo largo de la cual ya se había construido un túnel ferroviario, se abandonó y luego se reutilizó para realizar la nueva Via Olimpica para los Juegos Olímpicos de Roma de 1960.

En esos mismos años de la Posguerra, se anunció el concurso para la finalización de la nueva estación Termini por medio de la construcción del edificio principal. El concurso fue ganado en 1949 ex-aequo por los dos grupos formados por Eugenio Montuori, con Leo Calini, y Annibale Vitellozzi, con Massimo Castellazzi, Vasco Fadigati y Achille Pintonello, a quienes se les encomendó la tarea de desarrollar conjuntamente un nuevo proyecto.

La nueva estación fue inaugurada en 1950, con motivo del Jubileo, y desde entonces se ha ido consolidando progresivamente como el principal nodo intermodal de Roma. De hecho, además de la línea ferroviaria Roma-Fiuggi, inaugurada ya en $1916^{8}$, en 1955 se inauguró la línea B del Metro, a la que se agregaron la línea A y la extensión de la línea B hasta Rebibbia en 1990, con el ramal hacia Montesacro en 2015, cuyo único punto de intersección es precisamente Termini.

\footnotetext{
6 "La storia urbanistica di Roma è tutta e soltanto la storia della rendita fondiaria, dei suoi eccessi speculativi, delle sue convenienze e complicità colpevoli. Giulio Carlo Argan, Alcalde de Roma, junio de 1988". Desde: Italo Insolera, (1993), Roma Moderna. Un secolo di storia urbanistica 1870-1970. Nuova edizione. Piccola Biblioteca Einaudi.

7 Sin embargo, debe recordarse que la solución propuesta por Mazzoni para la fachada de la nueva estación no satisfizo la parte superior de Ferrovie dello Stato (como las soluciones que intentó aprobar inmediatamente después), así que solo se construyeron las dos alas laterales a lo largo de Via Marsala y via Giolitti - todavia existentes.

${ }^{8}$ El ferrocarril Rome-Fiuggi tenía el término al lado de la antigua estación de Termini, demolido en 1939.
} 
Al mismo tiempo, con el fuerte crecimiento de la ciudad inmediatamente después de la guerra, en Roma aparecen, por un lado, los barrios del auge económico - y de la gran especulación de finales de los años Cincuenta y principios de los Sesenta, en gran parte realizados fuera de los límites del anterior Plan de 1931. Por el otro, las llamadas "Borgate", poblados en su mayoría abusivo y lejos del centro de la ciudad, construidos a lo largo de las principales carreteras y ferrocarriles para dar respuesta a la emergencia vivienda, efecto de la llegada de los nuevos habitantes.

El primer plan moderno de Roma se remonta al 1965, cuando estas dinámicas de asentamiento estaban bien establecidas.

El Plan de $1965^{\circ}$ es el plan de la movilidad automovilística, de las expansiones hacia el mar y del Sistema Direccional Oriental, todo conectado por una nueva gran red de carreteras, que nunca se realizó por completo. Se pensaba de esta manera que Roma podía competir con otras ciudades europeas, donde, sin embargo, ya había entendido la importancia de un sistema de transporte público eficiente para acompañar el crecimiento de la ciudad ${ }^{10}$.

La "nueva" Roma que resulta de esto está rodeada sustancialmente por una "mermelada de construcciones" (Cerasoli, 2008), una periferia-dormitorio, heterogénea y a menudo ilegal, que combina la ausencia de espacios públicos y lugares centrales con la condición de provisional, lo que acentúa el carácter de periferia, donde los gérmenes de la suburbanización hacen su camino. Una periferia caracterizada por carreteras que terminan en nada, casi siempre sin aceras, y por un sistema de transporte público ineficaz, debido al diseño incoherente de las calles y la muy baja densidad de población, que produce el aumento constante en los niveles de tráfico y contaminación - producido por la plétora de desplazamientos obligatorios para quienes viven y trabajan en estas áreas (Cerasoli, 2008).

A pesar de los intentos esporádicos de remediar lo que estaba sucediendo, en Roma los vínculos entre planificación y gestión de la ciudad se debilitan cada vez más.

En la fecha de aprobación del Plan actual, en 2008, Roma parece afectada por la dispersión de los asentamientos y por la anomalía genética de las ciudades italianas mencionadas por Campos Venuti.

El Plan 2008 entonces propone una estrategia para la movilidad e las infraestructuras mejor conocida con el eslogan de la "cura del hierro", con el objetivo de integrar y mejorar el transporte público por ferrocarril, dejando un papel secundario a la movilidad del automóvil. Por este motivo, se firma un Acuerdo entre el Municipio de Roma y Ferrovie dello Stato, la Región del Lazio y la Provincia de Roma, gracias al cual se activa el servicio de Ferrocarriles Metropolitanos, que une el Municipio de Roma y los Municipios del área metropolitana.

\footnotetext{
${ }^{9}$ EI PRG de 1965 también incluyó el cierre del anillo de tren, en una derrota más exterior de la del 1931, que pero fue parcialmente realizado y finalmente activado para la Copa Mundial de Fútbol de 1990, en el tramo comprendido entre la "Estación San Pietro" y las nuevas estaciones "Olímpico" y "Vigna Clara"; las dos nuevas estaciones solo funcionarán durante el evento de la Copa Mundial, y solo para trenes especiales para los partidarios, cerrando definitivamente en 1992, dejando la línea nuevamente en abandono hasta hoy.

${ }^{10}$ Esta política, por ejemplo, está presente en el Plan de Cinco Dedos de Copenhague de 1947.
} 
Retomando las disposiciones del Plan de 1965, el Plan de 2008 vuelve a presentar el proyecto "Cierre del Anillo ferroviario al Norte - Nodo de intercambio Tor di Quinto", especializando la mayor parte del nodo ferroviario de Roma para el servicio de pasajeros llevado a cabo por las líneas regionales y metropolitanas.

El cinturón ferroviario es uno de los cinco proyectos estratégicos del Plan $2008^{11}$, que afectan a aquellas partes de la ciudad a las que se le asigna un papel estratégico y estructurante para la mejora de los rastros morfológicos, para fortalecer las identidades y definir las redes de vías de movilidad sostenible.

Al definir el Cinturón ferroviario como un área de planificación estratégica, el plan le atribuye un reconocimiento morfológico y relacional, paralelamente a sus aspectos funcionales, reinterpretándolo como una nueva "linealidad equipada y calificada".

Pero este tramo - aún por construir - desde Vigna Clara hasta el nodo de Nuovo Salario, para el cual también debe construirse un nuevo puente en el Tíber, se encuentra en una condición de grave deterioro y, aún hoy, está ocupado por actividades ilegales, que, de hecho, han impedido aún más la implementación del proyecto y para que tuvo necesario poner en marcha un proceso de negociación.

La estructura ferroviaria de Roma es el resultado de décadas de políticas urbanas fragmentadas y gestión urbana cada vez más engorrosas.

Hoy en día el nodo de la Estación Termini es el nodo más congestionado, poco eficiente y progresivamente degradado de la Capital y continúa representando una barrera entre los barrios Esquilino, Castro Pretorio y San Lorenzo.

La vasta plaza frente a la estación, Piazza dei Cinquecento, no presenta las características de un espacio público de relación, desempeñando únicamente la función de gigantesco terminal para unas veinte líneas de autobuses y dos líneas de tranvía. Un (no)lugar caótico, de paso y espera, degradado y causa de degradación en un Centro Histórico igualmente congestionado.

En 2011, para cambiar la situación, la construcción de la nueva Estación Tiburtina tenía como objetivo la descentralización de los trenes de alta velocidad, aligerando la Estación Termini. Pero, por el contrario, Ferrovie dello Stato decidió mantener la Alta Velocidad en Termini y también comenzar una serie de obras para expandir la misma estación (como la nueva Terraza comercial y el nuevo estacionamiento, ahora en construcción avanzada), condenando así a la recién nacida Estación Tiburtina a un declive inexorable.

Diez años después de la aprobación del Plan 2008, la situación de movilidad en el territorio romano es preocupante.

Hoy en día alrededor del 30\% de la población romana vivir fuera del GRA, el Grande Raccordo Anulare $^{12}$, en una ciudad cada vez más extendida y fragmentada, casi el doble en comparación

\footnotetext{
${ }^{11}$ Además del cinturón ferroviario, son los ámbitos: Mura; Flaminio-Fori-Eur; Tevere; Parco archeologico-monumentale dei Fori y dell'Appia Antica.

12 || Grande Raccordo Anulare es el anillo de autopista que rodea la ciudad consolidada y que construyo entre el 1951 y el 1970 .

Citación: CERASOLI, M. y AMATO, CH. Remendando la estación Termini. En: Libro de proceedings, CTV 2018. XII Congreso Internacional Ciudad y Territorio Virtual. "Ciudades y Territorios Inteligentes". Mendoza, 5-7 septiembre 2018. Barcelona: CPSV, 2018, p. 227-246. 
con hace solo veinte años. Esto hace cada vez más difícil garantizar un transporte público adecuado en una ciudad cada vez menos densa.

Por otro lado, ante la ausencia de una política de vivienda eficaz, el mercado inmobiliario ha empujado a muchos ciudadanos, generalmente familias jóvenes, a buscar casa en áreas fuera del GRA y en los municipios que rodean con Roma, generando una categoría de romanos, viajeros diarios, que demoran mucho más tiempo para ir al trabajo que los que viven dentro del GRA y generalmente tienen pocas alternativas al vehículo privado para hacer sus desplazamientos. En este panorama, un replanteamiento integral del nodo ferroviario de Roma es necesario y prioritario, a partir de una reflexión sobre su corazón, la Estación Termini.

\section{Figura 3. Nodo ferroviario de Roma}

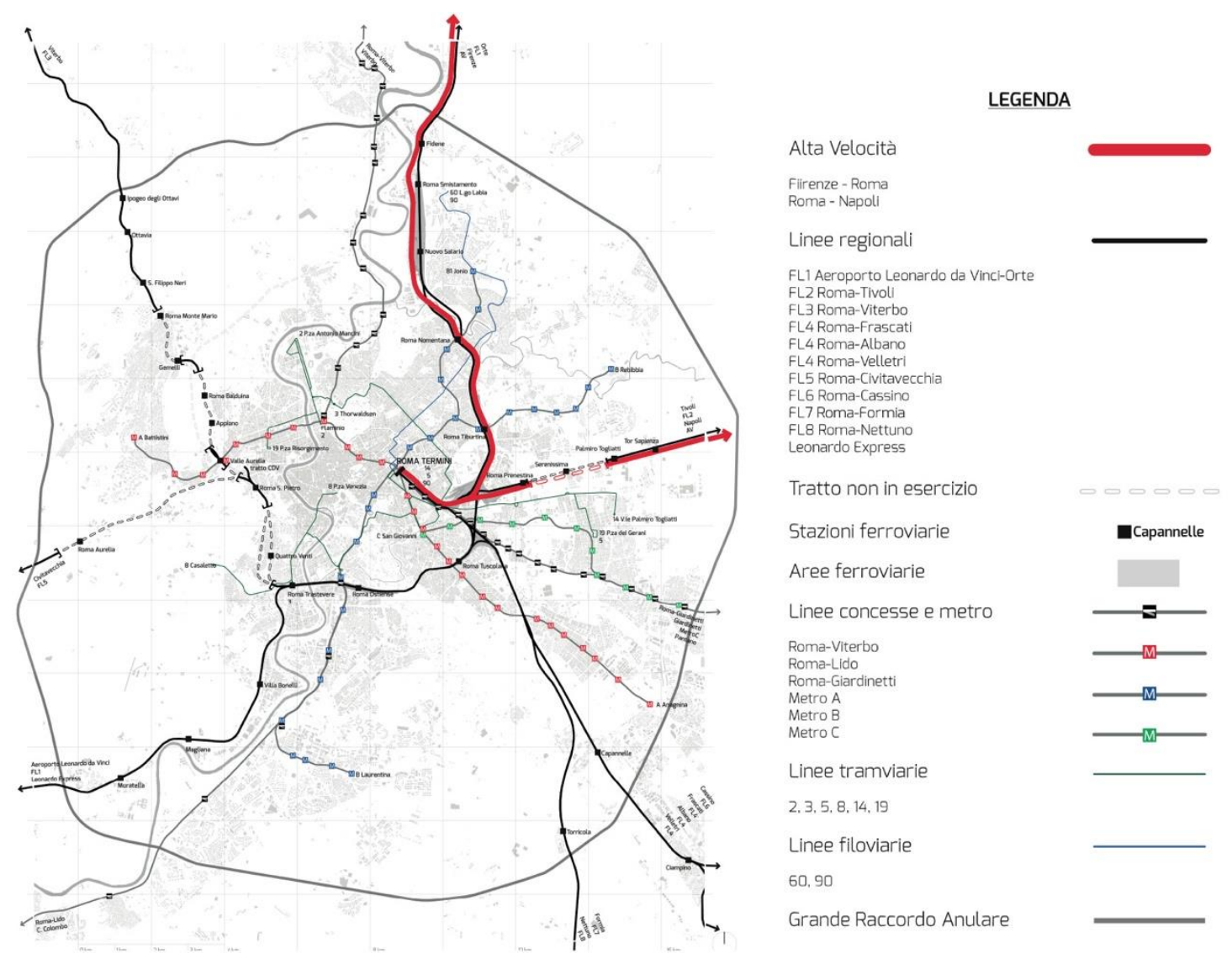

Fuente: Estudio desarrollado por los Dr. Arturo Becchetti [arturo.becchetti@gmail.com] y Dr. Fabrizio Felici [fabriziofx2@gmail.com], 2018. Universidad Roma Tre.

\section{El escenario de Mending Termini Station}

El escenario "Mending Termini Station" se basa en el proyecto para la Estación de Termini del Plan de 1931, que, actualizado y modificado adecuadamente, se vuelve a proponer con el objetivo, por un lado, de definir un sistema de movilidad integrado entre ferrocarriles, subterráneos, tranvías y transporte público de superficie, y, por otro lado, de iniciar un proceso de "remiendo" del centro de la ciudad de Roma.

Citación: CERASOLI, M. y AMATO, CH. Remendando la estación Termini. En: Libro de proceedings, CTV 2018. XII Congreso Internacional Ciudad y Territorio Virtual. "Ciudades y Territorios Inteligentes". Mendoza, 5-7 septiembre 2018. Barcelona: CPSV, 2018, p. 227-246. 
Confirmando el cierre del Anillo ferroviario y la construcción de una nueva estación en Tor di Quinto, el Escenario reitera la eliminación del área ferroviaria Termini actual, reemplazada por un pase ferroviario subterráneo norte-sur, dedicado exclusivamente al Alta Velocidad, y una nueva estación de AV "Termini", también subterránea.

Todas las estaciones del Anillo ferroviario, en las que se desviarían las líneas ferroviarias metropolitanas y regionales, actuarían como nodos de intercambio con otros sistemas de transporte público (metros, tranvías y trolleybuses) y, al mismo tiempo, con los principales colectores viales metropolitanos, garantizando así ese papel fundamental en la conexión entre ciudad y territorio que las estaciones siempre han tenido.

Termini se liberaría así del flujo constante de viajeros diarios y turistas (casi 500,000 por día ${ }^{13}$ ), convirtiéndose en una "puerta de entrada" al centro de Roma solo para viajeros del Alta Velocidad (menos de 100,000 por día ${ }^{14}$ ).

Figura 4. Mending Termini Station, nodo ferroviario de Roma
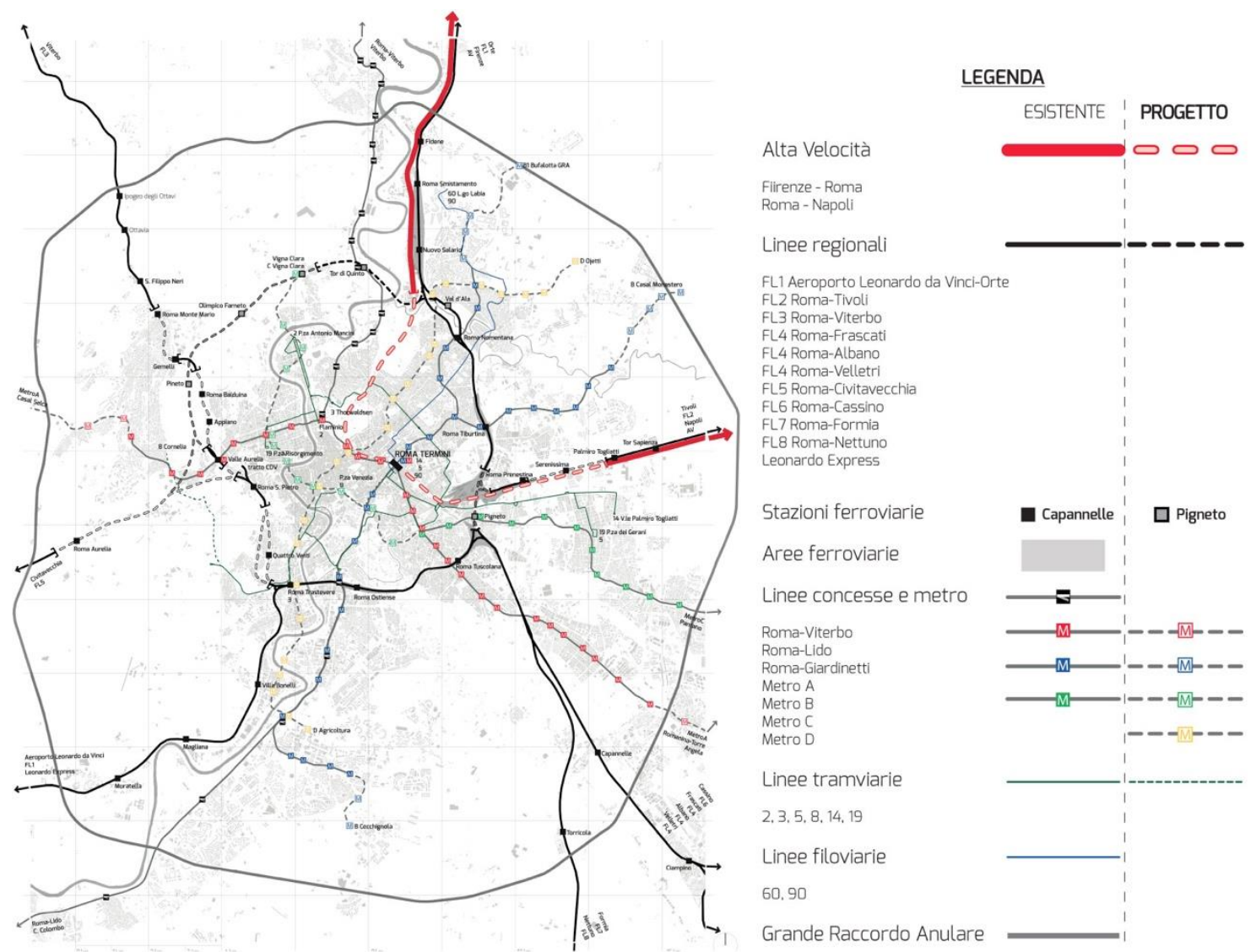

Fuente: Estudio desarrollado por los Dr. Arturo Becchetti [arturo.becchetti@gmail.com] y Dr. Fabrizio Felici [fabriziofx2@gmail.com], 2018. Universidad Roma Tre.

El Escenario también sería una oportunidad única para redefinir un área enorme en el corazón de la ciudad de Roma, comenzando un proceso de regeneración integral para recuperar la

\footnotetext{
${ }^{13}$ Fuente: Ferrovie dello Stato Italiane, 2018.

${ }^{14}$ Estimación de datos RFI, 2018.
}

Citación: CERASOLI, M. y AMATO, CH. Remendando la estación Termini. En: Libro de proceedings, CTV 2018. XII Congreso Internacional Ciudad y Territorio Virtual. "Ciudades y Territorios Inteligentes". Mendoza, 5-7 septiembre 2018. Barcelona: CPSV, 2018, p. 227-246. 
calidad urbana, ambiental y del transporte. En este sentido, el proyecto de reutilización de la Estación Termini, su área y, en consecuencia, su entorno debe abordar los problemas derivados de lo que es, de hecho, un fragmento urbano. Por lo tanto, se trata de priorizar los temas de permeabilidad, mixité funcional, calidad urbana y el remiendo de esta parte de la ciudad con las redes ambientales, de transporte e también intangibles presentes.

No debe olvidarse que, al lado del área de Termini, hay algunos lugares importantes en transformación, como las ex Aduana de San Lorenzo, una de las áreas de transformación proporcionadas por el Plan de 2008, y la Tangencial Este, una autopista urbana parcialmente elevada cuyo futuro aún está situado entre la demolición y la reutilización como greenway.

Los mismos barrios que rodean Termini ya están experimentando diferentes procesos de transformación: de turistificación masiva, en la parte más central de los barrios Esquilino y Monti; de gentrificación, en la parte más antigua de Pigneto, y de reemplazo de los habitantes originales, en la "Chinatown" alrededor de Piazza Vittorio (barrio Esquilino).

Finalmente, el Centro Histórico de Roma y, en particular, el área actualmente fuera de la ZTL Zona de Tráfico Limitado "Centro Histórico" pero situada dentro de las antiguas murallas de la ciudad - y por lo tanto patrimonio de la UNESCO - se necesita una nueva estrategia de movilidad, en línea con las otras capitales europeas, que preserva el patrimonio del esmog y del aparcamiento salvaje y que redefine morfológicamente y funcionalmente los ejes históricos y la huella de la ciudad.

A nivel urbano, los objetivos establecidos por Mending Termini Station toman nota del carácter multi-sistémico de los lugares y proponen la reparación de las fracturas urbanas producidas por el nodo de Termini, la inversión de la jerarquía de los modelos de movilidad, la protección del patrimonio histórico y arquitectónico y la integración de la red histórico-ambiental, a través de acciones tangibles e intangibles para una reconfiguración inteligente de lugares.

En cuanto al sistema de movilidad, con el cierre del Anillo ferroviario y la sustitución de la estación Termini actual con una estación subterránea solo para trenes de Alta Velocidad, la reorganización urbana prevé la reconfiguración de las ZTL Zonas de Tráfico Limitado: la ZTL "Centro Histórico" se extendería a todo el Centro Histórico definido por las murallas antiguas, sin límite horario; la ZTL "Anillo Ferroviario", mientras mantiene su extensión inalterada, se cobraría con un boleto diario para no residentes; la ZTL "Cinturón verde" inalterada.

Con respecto al transporte público, el Escenario prevé la integración de los tranvías existentes con una serie de extensiones y nuevas líneas, fortaleciendo las conexiones radiales desde el centro de Roma hasta el Anillo ferroviario, y el uso de minibuses eléctricos en la parte central e histórica de la ciudad.

Esta nueva estructura de movilidad permitiría ampliar las áreas peatonales existentes e integrarlas con nuevas. Finalmente, las intervenciones de reconfiguración de los ejes viales principales permitirían aumentar y sistematizar las áreas seguras para los peatones y las ciclo vías.

En cuanto al tejido construido, las intervenciones de regeneración urbana sugeridas por Mending Termini Station se dividen en siete Áreas de Recuperación y cuatro Zonas de

Citación: CERASOLI, M. y AMATO, CH. Remendando la estación Termini. En: Libro de proceedings, CTV 2018. XII Congreso Internacional Ciudad y Territorio Virtual. "Ciudades y Territorios Inteligentes". Mendoza, 5-7 septiembre 2018. Barcelona: CPSV, 2018, p. 227-246. 
Reestructuración Urbana. Las primeras incluyen los barrios San Lorenzo, Castro Pretorio, Termini, Esquilino, Casilino y Pigneto, para los cuales se especifican las categorías de intervención permitidas, así como las medidas específicas de rehabilitación y mejora; las segundas, en cambio, especifican la transformación de la antigua Estación Termini, las antiguas Aduanas de San Lorenzo, el área ferroviaria de Termini (a desmantelar) y la Tangencial Este.

Figura 5. Mending Termini Station, masterplan

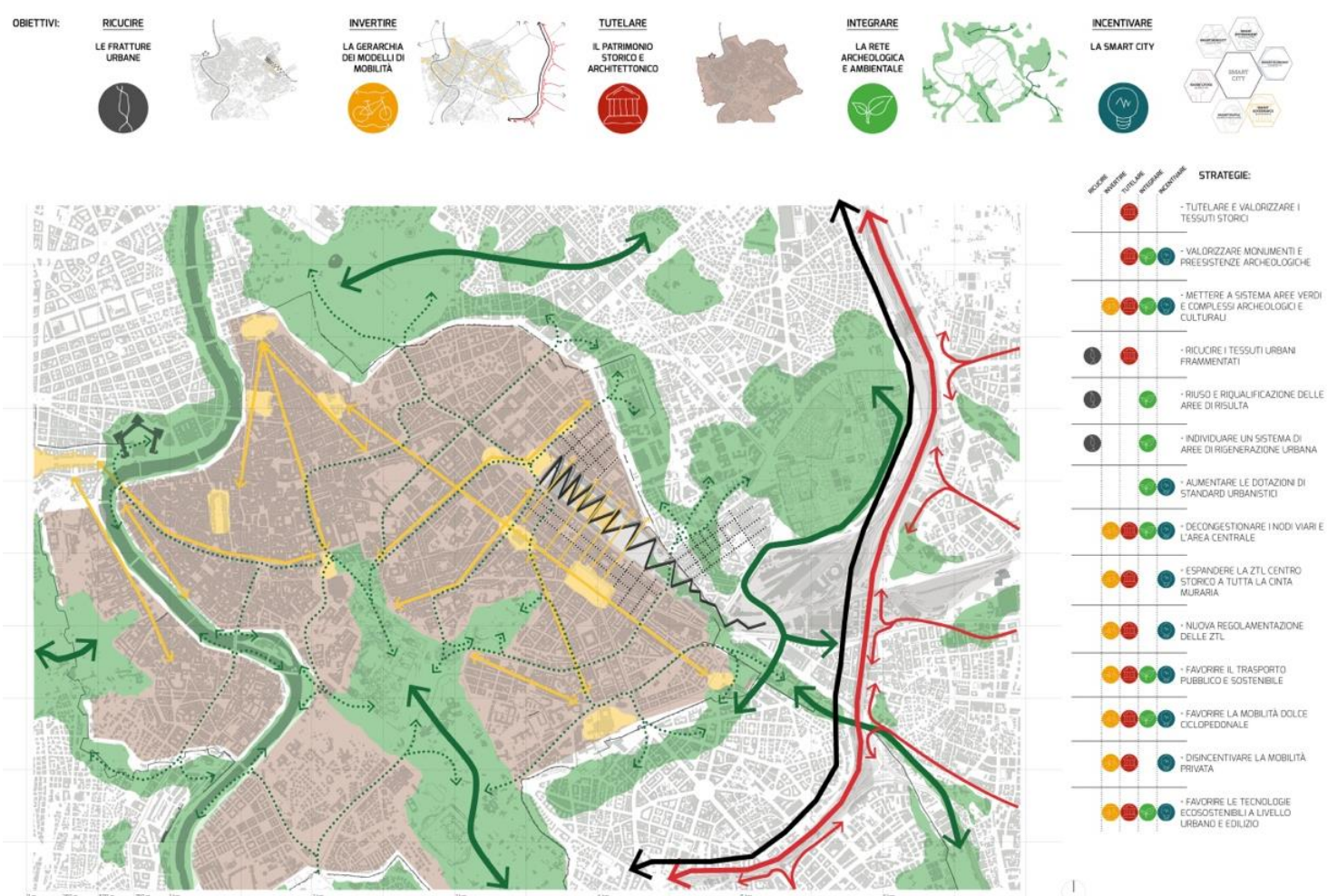

Fuente: Estudio desarrollado por el Dr. Arturo Becchetti [arturo.becchetti@gmail.com] y el Dr. Fabrizio Felici [fabriziofx2@gmail.com], 2018. Universidad Roma Tre.

La transformación más significativa es la que afecta al área ferroviaria de la Estación Termini, donde se planifican los nuevos edificios y el diseño general del área.

La antigua estación (diseñada por Montuori, Vitellozzi y Mazzoni), protegida y de enorme valor histórico y arquitectónico, se re-funcionaliza con usos mixtos, tanto públicos como privados, de acuerdo con los otros componentes de la intervención.

En el área entre las alas de la antigua estación, la parte más cerca del edificio principal, con vista a la Piazza dei Cinquecento, está destinada a servicios públicos y comercio; el área inmediatamente adyacente, a residencias especiales (viviendas para estudiantes y sociales), con el fin de garantizar la mezcla social.

El área central y más importante del área ferroviaria es para uso residencial, con actividades comerciales y servicios en la planta baja de los edificios; Por último, una zona verde pública equipada. Toda la zona es desprovista de automóviles, está completamente peatonal y está servida por un tranvía (la línea Termini-Centocelle, desviada).

Citación: CERASOLI, M. y AMATO, CH. Remendando la estación Termini. En: Libro de proceedings, CTV 2018. XII Congreso Internacional Ciudad y Territorio Virtual. "Ciudades y Territorios Inteligentes". Mendoza, 5-7 septiembre 2018. Barcelona: CPSV, 2018, p. 227-246. 
Desde el punto de vista morfológico, la intervención garantizará la permeabilidad del tejido construido y la accesibilidad, diseñando el sistema de espacios públicos de acuerdo con el contexto unitario en el que se insertarán y la naturaleza del lugar.

Finalmente, en cuanto al sistema histórico-ambiental, el objetivo general del Escenario es proporcionar a toda el área más espacios verdes, contribuyendo a la mejora medioambiental de los barrios vecinos. El Escenario contempla la recalificación y la reconexión de los espacios abiertos y verdes existentes y la construcción de una red ecológica que consta de cuatro nuevos parques:

- el Parque lineal "Ex-tangenziale", que utiliza la estructura elevada de la ex Tangencial Este, liberada del tráfico vehicular, definiendo una "highline" que pasa por alto también la zona de la Antigua Aduana de San Lorenzo;

- el Parque lineal "Anillo ferroviario", que se extiende a lo largo del ferrocarril, que se enterrará (por el Plan de 2008) y que a lo largo de su camino conecta las áreas verdes circundantes;

- el Parque central de Termini, que afecta al área ferroviaria que ya no se utilizará, hasta llegar al Parco degli Acquedotti;

- el Parque Lineal Integrado de las Murallas Aurelianas (por el Plan de 2008), que entra en conexión con los grandes parques y las zonas verdes más pequeñas vecinas, además de intervenciones puntuales en relación con las emergencias históricas.

\section{Factibilidad y actuaciones}

El escenario Mending Termini Station es una experimentación proyectual que representa una operación urbana compleja y participativa, que garantizaría la producción de una cantidad significativa de "ciudad pública", la reconexión e integración de los barrios vecinos y la creación de servicios públicos.

Los siguientes actores estarían involucrados:

- Grupo "Ferrovie dello Stato Italiane" (RFI Red Ferroviaria Italiana; Grandi Stazioni; FS Sistemi Urbani);

- Municipio de Roma;

- Región Lazio y Estado.

El escenario contempla la transformación del área ferroviaria actual (20 hectáreas), en una porción de la cual (35\%) RFI y FS Sistemi Urbani podrán realizar unas nuevas construcciones con varios usos (residencial, terciario, direccional).

El 65\% restante se cederá al Municipio de Roma para la construcción de servicios y parques. Al mismo tiempo, el edificio histórico de la Estación Termini, restaurado, estará disponible para ser rehabilitado y utilizado para diversos usos, públicos y privados.

RFI, con el apoyo del Estado y la Región Lazio, se haría cargo de la construcción del nuevo paso ferroviario subterráneo y de la nueva estación AV Termini también subterránea. 
La Municipalidad de Roma se haría cargo de la reorganización general de la movilidad y del mantenimento de los parques y de las áreas públicas.

La viabilidad se garantizaría mediante un procedimiento de negociación entre las dos partes principales involucradas, RFI, titular de los derechos de propiedad de las áreas, y el Municipio de Roma, titular del derecho a la transformación urbana.

El Acuerdo de Planificación y la Planificación participativa serían las herramientas para garantizar un proyecto de calidad, compartido, inclusivo y sostenible y la implementación contextual de todas las acciones complementarias (planes, proyectos, programas, etc.) necesarias para la mejora general del área central de Roma y, concretamente, la movilidad urbana y metropolitana.

Mending Termini Station, sin embargo, no es una utopía, ni es simplemente un ejercicio académico, sino una prueba de la posibilidad concreta de iniciar un proceso de regeneración urbana "inteligente", en el que el resultado positivo estaría representado por la gestión colegial y transparente de la ciudad y sus transformaciones.

\section{Bibliografía}

CAMPUS VENUTI, G; OLIVA, F; Cinquant'anni di urbanistica in Italia 1942 - 1992, Roma-Bari, Editori Laterza, 1993

CAMPUS VENUTI, G. II Sistema della Mobilità. En: Urbanistica, 2001, n. 116, 166-172 p.

CERASOLI, M. Periferie urbane degradate. Roma, la direzione verso il mare, Napoli, Edizioni Scientifiche Italiane, 2009, 885 - 895 p.

CERASOLI, M. Politiche ferroviarie, modelli di mobilità e territorio. Le ferrovie italiane nell'epoca della pseudo-liberalizzazione. Roma, Aracne Editrice, 2012

CERASOLI, M. L'annosa questione del Nodo di Roma. En: Urbanistica Informazioni, n. 249 250, 2013, 14-16 p.

CERASOLI, M., Qualità urbana, mobilità, qualità della vita: una "grammatica" per il Rinascimento della città. In: Urbanistica Informazioni, $n^{\circ} 263$ S.I., sessione speciale $n^{\circ} 7$, pag. 16-19. ISSN: 0392-5005

CERASOLI, M; AMATO C.; Mending Termini Station, una utopia per la Città Eterna, En: Urbanistica Informazioni, n. 278, 2018, 24-29 p.

PUCCI, P. Liberalizzazione nel settore ferroviario ed effetti sulle politiche di trasformazione fondiaria. Il caso degli scali ferroviari a Milano. In: CERASOLI, M., Politiche ferroviarie, modelli di mobilità e territorio. Le ferrovie italiane nell'epoca della pseudo-liberalizzazione. Roma, Aracne Editrice, 2012, 68 - 80 p.

Citación: CERASOLI, M. y AMATO, CH. Remendando la estación Termini. En: Libro de proceedings, CTV 2018. XII Congreso Internacional Ciudad y Territorio Virtual. "Ciudades y Territorios Inteligentes". Mendoza, 5-7 septiembre 2018. Barcelona: CPSV, 2018, p. 227-246. 
INDOVINA, F., Dalla città diffusa all'arcipelago metropolitano. Milano, FrancoAngeli, 2009

INSOLERA, I., Roma Moderna. Un secolo di storia urbanistica. 1870-1970, Torino, Einaudi, 1993

ROSSI, P. O. Roma. Guida all'architettura moderna 1909-1991. Bari, Laterza, 1991 